\title{
A young woman presenting a kikuchi-fujimoto's disease associated with induced lupus
}

\author{
Galith Kalmi1,2, Karine Champion1, Sarah Lechtman ${ }^{1,2}$, Stéphane Mouly1,2, Damien Sène ${ }^{1,2^{*}}$
}

\begin{abstract}
${ }^{*}$ Correspondence
Damien SENE

Department of Internal Medicine, Hôpital Lariboisière, 2 rue AmbroiseParé, 75010 Paris, France Email: damien.sene@aphp.fr

Phone: +33-149956393

${ }^{1}$ Department of Internal Medicine, Hôpital Lariboisière, Paris F-75010, France

2 Paris Diderot University, Paris, France

Received: Jul 16, 2018

Accepted: Nov 5, 2018

Published: Nov 23, 2018

(c) Galith Kalmi et al, 2018; licensee OA Journal of Case Reports. This is an Open Access article distributed under the terms of the Creative Commons Attribution License (http:// creativecommons.org/licenses/by/4.0) which permits unrestricted use, distribution, and reproduction in any medium, provided the original work is properly credited.
\end{abstract}

\begin{abstract}
Introduction: Kikuchi-Fujimoto's disease (KFD) or histiocytic necrotizing lymphadenitis is a benign and self-limited disease of unknown etiology mainly affecting young women. Although the association with systemic lupus erythematosus (SLE) is well described, no case of drug-induced lupus erythematosus (DILE) associated KFD has not been reported so far.

Case report: We herein report a 25-year old Caucasian woman, with no medical history and no medication except for oral estrogenprogestin contraception (levonorgestrel-ethinylestradiol), who presented with cervical lymphadenopathy, fever and arthralgia without weight loss, night sweats or skin involvement. An exhaustive infectious disease screening was negative and lymph node biopsy revealed histiocytic necrotizing lymphadenitis suggesting KFD. Autoimmune screening tests evidenced high titers of anti-histone antibodies suggesting DILE induced by estrogen-progestin medication. The patient received a short course of non-steroidal anti-inflammatory treatment for painful lymphadenitis and arthralgia. Oral levonorgestrel-ethinylestradiol contraceptive medication was stopped and KFD and DILE completely recovered with a long-term disappearance of anti-histone antibodies.

Conclusion: We report the first case of KFD associated-DILE following oral levonorgestrel-ethinylestradiol medication. Even though levonorgestrel-ethinylestradiol induced lupus is well known, the
\end{abstract} association with KFD has never been reported and the physiopathology remained unknown.

Keywords: Kikuchi-Fujimoto disease, Lupus, Levonorgestrel-ethinylestradiol

\section{Introduction}

Kikuchi-Fujimoto's disease (KFD) or histiocytic necrotizing lymphadenitis is a rare, benign and selflimited disease revealing itself by cervical lymphadenopathy and high-grade fever, mainly affecting young women. It was first described in 1972 by Kikuchi and Fujimoto. The diagnosis is made by lymph node histology: histiocytic necrotizing lymphadenitis which is characterized by patchy areas of necrosis, with eosinophilic materials and nuclear debris and the absence of neutrophils $[1,2]$.
The etiology is still unknown butviral etiologysuch as Epstein Barr virus (EBV) [3] or Human Herpes virus 7 (HHV7) [4] have been proposed. This disease can be isolated or associated with systemic diseases, in particular with systemic lupus erythematosus (SLE), preceding, coexisting or following the diagnosis $[\underline{5}, \underline{6}]$. The main differential diagnosis are infectious diseases and lymphomas.

Drug-induced lupus erythematosus (DILE) is a variant of lupus erythematosus that resolves within days to 
months after withdrawal of the drug in a patient with no underlying immune system dysfunction. DILE can arise months to years after exposure to the drug. The most common drugs that cause DILE are hydralazine, procainamide, quinidine, isoniazid, diltiazem, and minocycline []].

Although the association with systemic lupus erythematosus is well described $[\underline{5}, \underline{6}]$, no case of drug-induced lupus erythematosus associated KFD has not been reported so far.

We report the case of a KFD preceding a DILE.

\section{Case Report}

We herein report a 25-year old Caucasian woman, with no medical history and no medication except for oral estrogen-progestin contraception (levonorgestrel-ethinylestradiol called Minidril ${ }^{\circledR}$ ), who presented with cervical lymphadenopathy, fever and arthralgia without weight loss, night sweats or skin involvement.Blood analysis revealed normal blood cells count (leukocytes $5200 / \mathrm{mm} 3$, hemoglobin $12.4 \mathrm{~g} / \mathrm{dL}$, platelets $258000 / \mathrm{mm} 3$ ) liver. Serum complement C3and C4 levels were $1.23 \mathrm{~g} / \mathrm{L}$ (Normal range $0.8-1.8 \mathrm{~g} / \mathrm{L}$ ) and $0.16 \mathrm{~g} / \mathrm{L}$ (Normal range $0.15-0.45 \mathrm{~g} / \mathrm{L})$, respectively. The $\mathrm{C}$ - reactive protein (CRP) level was $10 \mathrm{mg} / \mathrm{L}$ (Normal range < $3 \mathrm{mg} / \mathrm{l}$ ).
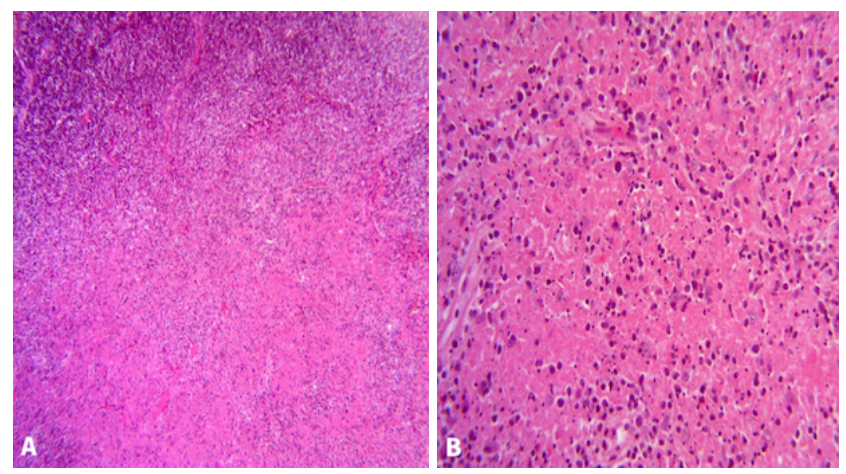

An exhaustive infectious disease screening was negative in particular HIV, CMV, EBV, toxoplasmosis, HHV6, HHV7, HHV8, parvovirus B19, brucellosis serology. A whole-body scanner only showed the known cervical lymphadenopathy. The lymph node biopsy revealed histiocytic necrotizing lymphadenitis (figure 1), suggesting diagnosis of KFD. Autoimmune screening tests were realized: antinuclear, native-anti DNAs, and anti ENA antibodies were negative but anti-histone titer was borderline $(26 \mathrm{UI} / \mathrm{L})$. The patient revised 3 months later with persistence of cervical lymphadenopathy and arthralgia. A new autoimmune screening was realized and found positive native anti-DNA antibodies (88UI/L) and positive anti histone (>300UI/L) evoking strongly a drug-induced lupus erythematosus. The patient received a short course of non-steroidal antiinflammatory treatment for painful lymphadenitis and arthralgia and her only long-term treatment, oral estrogen-progestin contraception, was stopped.

Cervical lymphadenopathy and arthralgia had completely disappeared 6 months later. Anti-DNA and anti-histone antibodies and CRP were also negative. KFD and DILE completely recovered after levonorgestrel-ethinylestradiol withdrawal with a long-term disappearance ofanti-DNAs and antihistone antibodies.

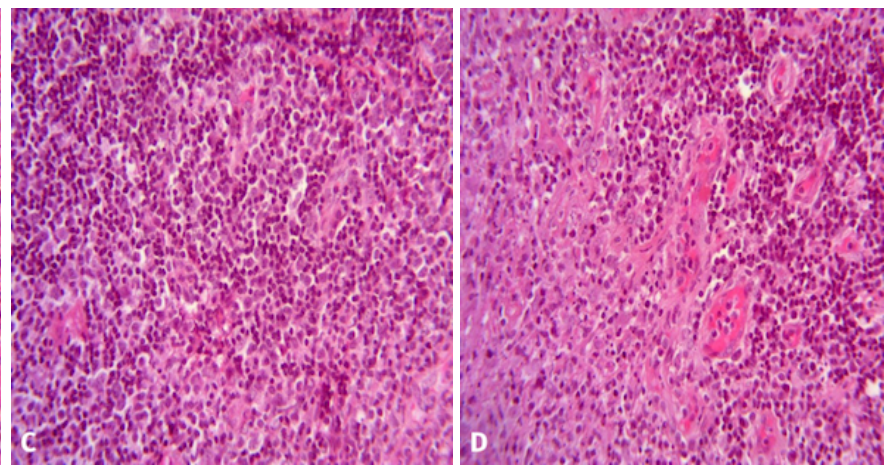

Figure 1: Wide areas of histiocytic infiltrate with prominent necrosis (hematoxylin and eosin). (A) X4 general view with normal lymph node and necrosis (B) X 16 necrosis (C) X16 normal lymph node (D) X16 junction normal lymph node and necrosis

\section{Discussion}

The association between KFD and SLE is well known in medical literature but the association with DILE has not been described. We relate, to our knowledge, the first case of DILE associated with KFD, with a regression of symptoms and biological anomalies after the suspected drug was stopped. Even though levonorgestrel-ethinylestradiol induced lupus is well known [요 9 ], KFD occurring after this treatment has not been reported.

However, the possibility of coexisting DILE and KFD occurred by chance cannot always be excluded in this case. 
SLE presenting with KFD as a long-term sequela of Drug-Induced Hypersensitivity Syndrome (DIHS) has been reported [10]. DIHS is a severe form of drug eruption associated with viral reactivations. Autoimmune diseases have been described to develop several months or years after the resolution of DIHS. N. Aota et al [10] have reported a case of a woman developing a SLE with KFD 4 years after an episode of DIHS associated with human herpesvirus 6 (HHV6) and EBV reactivation. Carlson JA et al [11] have reported a DIHS after antibiotic associated with EBV replication revealing KFD like histology. These reports suggest a close link between drug-induced KFD associated or not with SLE and EBV reactivation. For these patients, DILE associated with KFD has not been addressed and autoimmune screening in particular anti-histone antibodies has not been realized.

An association between KFD and DILE with a mediation by EBV reactivation is possible but, in our case, there was no associated EBV reactivation.

\section{Conclusion}

In conclusion, we report the first case of KFD associated-DILE following oral levonorgestrelethinylestradiol medication. Even though levonorgestrel-ethinylestradiol induced lupus is well known, the association with KFD has never been reported and the physiopathology remained unknown. An autoimmune screening with antihistone antibodies is essential.

\section{References}

1. Adjaoud D, Boudjemaa S, Boccon-Gibod L et al. (2007) Maladie de Kikuchi-Fujimoto: à propos de 5 cas et revue de la littérature. Archives de Pédiatrie. 14: 1333-1336.

2. Astudillo L (2010) Kikuchi-Fujimoto disease. Rev Med Interne 31: 757-765.

3. Lee HY, Huang YC, Lin TY, et al. (2010) Primary Epstein-Barr virus infection associated with Kikuchi's disease and hemophagocytic lymphohistiocytosis: a case report and review of the literature. J Microbiol Immunol Infect. 43: 253-257.

4. Labrador J, Aparicio MA, Santos-Briz A, et al. (2013) KikuchiFujimoto disease: a case supporting a role for human herpesvirus 7 involvement in the pathogenesis. Rheumatol. Int. 33: 3065-3068.

5. Santana A, Lessa B, Galrão L, et al. (2005) Kikuchi-Fujimoto's disease associated with systemic lupus erythematosus: case report and review of the literature. Clinical Rheumatology 24: 60-63.

6. Chen HC, Lai JH, Huang GS, et al. (2005) Systemic lupus erythematosus with simultaneous onset of Kikuchi-Fujimoto's disease complicated with antiphospholipid antibody syndrome: a case report and review of the literature. Rheumatology International 25: 303-306.

7. Chang C, Gershwin ME (2011) Drug-induced lupus erythematosus: incidence, management and prevention. Drug Saf. 34: 357-374.

8. Gueli N, Toto A, Tartaglione E, et al. (1987) Systemic lupus erythematosus during therapy with oral estrogen-progestin. Considerationsapropos of a case. Clin Ter. 121: 299-304.

9. Kawai ., Katoh K, Matsunaga K., et al. (1989) A case of systemic lupus erythematosus after taking oral contraceptives. Nippon NaikaGakkaiZasshi. 78: 710-711.

10. Aota N, Hirahara K, Kano Y, et al. (2009) Systemic Lupus Erythematosus Presenting with Kikuchi-Fujimoto's Disease as a Long-Term Sequela of Drug-Induced Hypersensitivity Syndrome. Dermatology. 218: 275-277.

11. Carlson JA, Perlmutter A, Tobin E, et al. (2006) Adverse antibiotic-induced eruptions associated with epsteinbarr virus infection and showing Kikuchi-Fujimoto disease-like histology. Am J Dermatopathol. 28: 48-55. 